\title{
A Standard Model Approach to Inflation
}

\author{
Robert Bruce Hayes \\ Nuclear Engineering Department, North Carolina State University, Raleigh, NC, USA \\ Email: rbhayes@ncsu.edu
}

How to cite this paper: Hayes, R.B. (2022) A Standard Model Approach to Inflation. Journal of Modern Physics, 13, 113-121. https://doi.org/10.4236/jmp.2022.132009

Received: January 6, 2022

Accepted: February 11, 2022

Published: February 14, 2022

Copyright (C) 2022 by author(s) and Scientific Research Publishing Inc. This work is licensed under the Creative Commons Attribution International License (CC BY 4.0).

http://creativecommons.org/licenses/by/4.0/

\begin{abstract}
By assuming the cosmological principle includes the Pauli Exclusion Principle (PEP) and that the initial singularity existed within Planck time and length scales, a model for inflationary expansion is argued using only standard model physics without any changes to general relativity. All Fermionic matter is forced by the PEP to make a quantum transition to minimally orthogonal states in sequential Planck time intervals. This results in an initial inflation effect due to nearest neighbor quantum transitions which is then exacerbated by matter and antimatter creation effects due to collisions giving rise to the observational effects of universal inflation. The model provides a mechanistic explanation for primordial expansion using only physics from the standard model, specifically utilizing the PEP as a repulsion force between indistinguishable fermions. The present theory offers the benefit of not requiring any particles or fields outside of the standard model nor utilizing changes to general relativity. More succinctly, this theory goes beyond simply offering a mathematical representation (or fit) of the functional dependence but rather offers a mechanistic model to drive inflation using only standard model physics.
\end{abstract}

\section{Keywords}

Inflation, Cosmological Expansion, Standard Model, Pauli Exclusion Principle, Genesis

\section{Introduction}

The inflationary model has enjoyed great success in describing modern cosmological observations of homogeneity and isotropy along with a flat space-time [1] [2]. Difficulties with any mechanistic origin of the ad-hoc inflaton [3] have resulted in numerous alternative descriptions of the initial rapid expansion of the universe. These models include unique general relativity cases such as bouncing [3] [4] [5] [6], varying speed of light requirements [7] [8] [9], string theory [10] 
[11] along with multiple other alternatives [12] [13] [14] [15]. Still, other models provide functional representations of both inflation and dark energy [16] [17] [18]. The current work diverts from the traditional functional representations of inflation and proposes an entirely mechanistic model such that only modern physics is sufficient to require inflationary origins.

The effect from going back in time predicted by general relativity requires that all matter began in a singularity without a sufficient time dependent cosmological constant to reverse the process early on [19]. By assuming the singularity, the proposed theory is able to describe a mechanism to initiate inflationary expansion at genesis. It is reasonable to assume continuity of all the known physical laws even at the Planck scales [20] [21] backwards in time to include this primordial singularity. Here, particular attention is placed on requiring the Pauli Exclusion Principle (PEP) to also be in full effect at the Planck scale from which the proposed mechanism is derived.

The minimum stable state for baryonic matter which can be associated with adhering to the PEP requirement is postulated to scale with that of either a neutron star (NS) or atomic nuclei. This means that to a first approximation, all indistinguishable Fermionic matter (i.e., quarks) which had been present in the big bang (BB) singularity are forced at a minimum to push their nearest neighbor Fermions away on the order of this maximum packing density for nucleonic matter. The principle being that by combining the fundamental assumption of existence at the Planck scale in the singularity, it can then be argued that PEP also applies at the Planck scale and forces minimal physical separation in one Planck unit of time as a standard quantum transition from one state to another.

Given that all leptons, quarks and baryons of the standard model are composed of fermions, the anisotropy of their respective wavefunctions forces the PEP to uniformly distribute them into their minimally orthogonal and lowest energy states upon existence with the quantum transition taking place over the assumed Planck time scale. This, because of overlap of identical antisymmetric wavefunctions would result in cancellation of some of the particle's probability density function and so violate conservation of lepton and baryon numbers resulting in an effective separation force to maintain conservation of lepton number [22].

One of the most fundamental observations arising from PEP in measurements, is the repulsive force it provides when placing materials under pressure. It is the PEP which keeps crystalline materials at fixed interatomic distances despite the Coulombic attraction between the oppositely charged particles. When two objects are pushed together, it is the PEP repulsive force which prevents the exterior valence electrons of the two objects from overlapping and so serves as the equal and opposite force to their being pushed together [23].

\section{Analysis and Results}

The standard FLRW metric given by Carroll et al. [24] is $H^{2}=\frac{8 \pi G}{3} \rho_{M}+\frac{\Lambda}{3}-\frac{k}{a^{2}}$ assuming $k=0$ describes the current model for universal expansion (where the 
standard symbol definitions apply i.e., $\left.H^{2}=(\dot{a} / a)^{2}\right)$. When the Hubble length a approaches zero, the proper time from general relativity $\mathrm{d} \tau^{2}=\mathrm{d} t^{2}-\mathrm{d} x^{2} \mathrm{~d} \Omega^{2}$ becomes ill defined where both the spatial component $\mathrm{d} x$ and the temporal component $\mathrm{d} t$ approach zero as the FLRW model matter density $\rho_{M}$ goes to infinity at $\tau=0=a$. Rather, we will assume that at the beginning of time, the BB singularity evolves at the Planck scale.

\subsection{Inflation}

\subsubsection{Expansion Initiation}

The initial singularity scaling $a \approx 0$ is taken to be on the order of the Planck length $l_{p}=1.6 \mathrm{e}-35 \mathrm{~m}=\hbar m_{p}^{-1} \cdot c^{-2}$ which is calculated using the definition of Planck mass $m_{p}=2.177 \mathrm{e}-8 \mathrm{~kg}=(\hbar c / G)^{1 / 2}$ where $c$ and $G$ have their customary definitions of the speed of light and the gravitational constant respectively. These assumptions are also taken to occur in the initial time interval of the Planck time $t_{p}=5.4 \mathrm{e}-44 \mathrm{~s}=l_{p} / c$ which then provides a means to predict the effects from the PEP to all fermionic matter at its genesis.

The scale assumed here for quark density is taken to be similar to that associated with a NS or barionic nuclei. The minimally orthogonal baryon number density of $0.16 \mathrm{fm}^{-3}[25]$ then provides some initial condition predictions after the passage of the first unit of Planck time. At zero time, we begin with any arbitrary number of fermions in the singularity.

\subsubsection{The $1^{\text {st }}$ Planck Time Interval}

The overlapping fermion wave functions in the initial singularity simply make a quantum transition to an adjacent location to conserve fermion number. This fundamentally accepts the assumption that the requisite PEP separation has to take place within a single interval of the Planck time and so allows a calculation of the momentum transfer imparted to fermionic matter due to its genesis.

Using the scaling from that of a neutron in a NS [25], the resulting relative displacement $L$ between nearest neighbors for each quark would then be $L \approx 2 \mathrm{e}-15 \mathrm{~m}$ in the initial time interval $\sim 5 \mathrm{e}-44 \mathrm{~s}$. This quantum transition then culminates in an apparent violation of special relativity as the initial relative speed of any two adjacent fermions becomes $V \sim 2 \mathrm{e}-15 \mathrm{~m} / 5 \mathrm{e}-44 \mathrm{~s} \approx 1 \mathrm{e} 20 c$ (although this is really just a simple quantum transition for each fermion in a single unit of Planck time). In this $1^{\text {st }}$ Planck interval, each fermion has just transitioned outside the horizon of its nearest neighbors making them no longer causally connected at that moment.

With the initial dimensions of fundamental particles assumed to be Planck length going to a nearest neighbor distance $L$ of $2 \mathrm{e}-15 \mathrm{~m}$, this provides an expansion of 20 orders of magnitude during that $1^{\text {st }}$ Planck time interval alone. With minimally orthogonal states being required, this initiates a homogenous, though hyper-chaotic, initial condition.

If these first generation quarks were the lowest energy state available upon existence, this means each bare quark mass can be approximated as $m \approx 5 \mathrm{MeV} / c^{2}$ [26]. The resulting kinetic energy $K E$ from the initial quantum transition of nearest neighbors can then be calculated from $\sqrt{p^{2} c^{2}+m^{2} c^{4}}-m^{2} c^{4} \approx p c$. Al- 
though relativistic values become imaginary at such speeds, being that this is really just a quantum transition, we will use for relative interparticle momentum, the value of $p=m v=1 \mathrm{e} 22 \mathrm{MeV} / c$, giving a contribution to the $K E$ per quark of 1 e22 MeV. This places the total energy of the transition more than 20 orders of magnitude greater than the initial rest mass per fermion.

This process is depicted in Figure 1 where reflecting boundaries are assumed on the rightmost portion of the image.

\subsubsection{The $2^{\text {nd }}$ Planck Time Interval}

Within this closed packed configuration from the $1^{\text {st }}$ Planck time interval, the subsequent fermions can also be assumed to obey the uncertainty principle $\Delta E \sim \hbar / \Delta t \approx 7 \mathrm{e}-22 \mathrm{MeV} \cdot \mathrm{s} / 5 \mathrm{e}-44 \mathrm{~s} \sim 1 \mathrm{e} 22 \mathrm{MeV}$ placing this kinetic energy component effectively equal to that caused by the PEP imposed on the existence criteria. This means the kinetic energy during the second Planck time is approximately equally divided between and expansion motion and random motion for all particles. This provides a convenient mechanism to insure effective thermal equilibrium at existence without the need for any empirical coupling between disjoint regions outside each other's horizon. To the extent that this homogeneity continues due to these random expansion forces, uncoupled macroscopic regions would evolve in a similar chaotic manner with virtually indistinguishable phase space distributions.

The combined expansion energy and random kinetic energy coupled with extreme closed packing then implies fermion collisions are taking place. In other words, minimal orthogonality means that adjacent particles are touching an expected neighbor but with spatial offsets to allow distinguishable quantum numbers for each fermion. The resultant extreme high energy impacts between adjacent fermions having an average of $1 \mathrm{e} 22 \mathrm{MeV}$ of kinetic energy will create a vast sea of matter and antimatter particles, bringing these into existence even when they were not required in the initial singularity.

\subsubsection{The $3^{\text {rd }}$ Planck Time Interval}

To a good approximation, the uncertainty principle energy alone is sufficiently large to assume equal fractions of massless and massive particles and antiparticles all being formed in subsequent Planck time intervals [27]. Those which were created in the $2^{\text {nd }}$ time interval would largely be annihilated in the $3^{\text {rd }}$ Plank time interval due to spatial overlap.

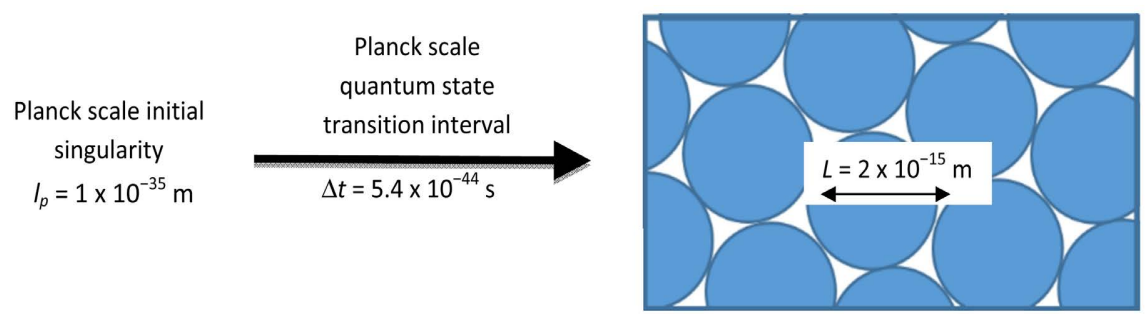

Figure 1. Quantum transition of a Planck scale singularity to a maximum packing density fermion sea. 
The remaining fermions from any prior Planck time interval would then be replaced by these new fermions which did not recombine with their antiparticles. Those in the current Planck time would again be subject to the same physics previously described and so would continue the process of creating new particles until the attractive forces are able to catch up to the kinetics of the ensuing rapidly evolving inflationary epoch.

In each Planck time interval, the number of residual remnant created Fermions would scale with the statistical fluctuations in the number of particles created in each volume. To provide an initial estimate of this value, assume the average standard model particle rest mass is $100 \mathrm{MeV}$ such that the $1 \mathrm{e} 22 \mathrm{MeV}$ energy per fermion is creating an additional $1 \mathrm{e} 20$ particles at each fermion location. The statistical fluctuations from this swarm of particles packed into this $0.16 \mathrm{fm}^{-3}$ would then be $1 \mathrm{e} 10$ additional fermions. These fermions which then remain unto the $4^{\text {th }}$ and further Plank time intervals would then undergo additional PEP quantum transitions exacerbating the inflationary process further. This process would continue through successive time intervals until attenuated by other means.

\subsubsection{Subsequent Planck Time Intervals}

The newly created particles with each prior Planck time interval (which were not annihilated by their antiparticles) will require PEP transitions which again will further exacerbate these inflationary effects.

After a time period of $L / c=2 \mathrm{e}-15 \mathrm{~m} / 3 \mathrm{e} 8 \mathrm{~m} \cdot \mathrm{s}^{-1} \sim 1 \mathrm{e}-23 \mathrm{~s}$ or approximately $3 \mathrm{e} 24$ Planck intervals, this is when gravity (and photons) will first start to appreciably be felt by nearest neighbors. By this time, each $0.16 \mathrm{fm}^{-3}$ of volume will have created approximately $1 \mathrm{e} 10$ fermions for every preceding Planck interval $(\sim 3 \mathrm{e} 24)$. Each new fermion then which had PEP transitioned to a new adjacent location then holds to the same rules as those prior, creating an average of $1 \mathrm{e} 10$ new fermions and so forth for an apparent runaway particle generation mechanism.

With each successive generation of particle creation giving rise to $1 \mathrm{e} 22 \mathrm{MeV}$ of new energy, each of these will send off gravitational waves along with their associated gluons, pseudoscalor and vector mesons to eventually provide adhesion forces. The photons will be able to undergo energy to mass transitions but not so for the gravitons as these would pass through each other as pure waves (consistent with the latest LIGO findings [28]). This means the gravity will continually build up and eventually be felt by adjacent and even distant newly created particles.

This gravitational pull will have been being built up by all preceding Planck interval particles and so by around 1e 25 intervals, it can be assumed that contraction forces would start to be felt. By this time, the number of created fermions alone would be on the order of (1e25)e10 or 1e250 fermions not to mention the associated melee of exotic particles in the mix. This number is approximately 120 orders of magnitude larger than that known in the observable un- 
iverse [29] and so scales how vast the physical universe may actually be outside of our current horizon.

It is generally accepted that all matter present today is an arbitrarily small fraction of this overabundance of matter compared to antimatter present in the initial mix, other conditions are possible [30] but these are all assumed here not to violate the PEP and so are fully consistent with the proposed model offered here for inflation.

\subsection{Isotropy and Homogeneity}

The spatial location of any fermion at any time is irrelevant to the inflationary mechanism proposed leaving their evolution in phase space as effectively identical as the forces are identical but chaotic. This means that despite the lack of causal connectedness, the model still leaves each region to evolve in identical conditions subject to deviations due to random motions and statistical variations. In this sense, the expected outcome is effectively a homogenous distribution on the large scale after local coalescing effects are taken into consideration. The massive inflationary expansion also explains the expected flatness of space on the large scale as the effective stretching from the initial chain reaction of particle production would have imposed this condition.

\section{Discussion}

Utilizing the PEP, the uncertainty principle and the conservation laws, sequential Planck time units for quantum transitions create a massive chain reaction of particle creation sufficient to explain inflationary origins. This model effectively places inflation at the very initial moments of the BB eventually ( 1e-20 s later) followed by random deceleration and cooling from subsequent attractive interactions. Standard BB cosmological models then continue to evolve using currently understood particle physics and general relativity models. This was accomplished using an arbitrary number of starting particles in the initial singularity.

The proposed inflation mechanism also has a certain elegance in that it only requires making almost intuitive and basic assumptions regarding initial existence at the Planck scale along with an axiomatic adherence to the PEP, from these, inflation is postulated effectively at genesis. Specifically, it is assumed that within the Planck time at the $\mathrm{BB}$ singularity, the PEP forces any arbitrary number of primordial adjacent like fermions apart sufficient to enable minimally distinct particle wavefunctions. The resultant energy from expansion and uncertainty momentum then creates a large number of other fermions whose remnant (which statistically did not recombine with antiparticles) then creates a subsequent generation of fermions to carry on the process. This continues until gluons, photons, and gravity (including the gravitational effect of neutrinos) can eventually start to coalesce the massive expansion forces.

This Pauli force effectively provides the initial starting energy of expansion by 
requiring all Fermions to have these distinct quantum states. All identical Fermions then start with all others outside their horizon with random motion driving subsequent evolution. This model accounts for why the universe is so smooth on large scales, the requisite minimally orthogonal states at the initial Planck time forces this to be the initial condition everywhere. Likewise, flatness is postulated to be due to a massive scale and a subsequent purely random walk in all directions of all particles preventing curvature on large scales while still allowing clumping due to the same mechanism on small scales.

Conservation of lepton and boson number can be obtained within each horizon for the initial inflationary period but is not addressed further in this work but certainly warrants future attention. That conservation laws are due to symmetries in nature as demonstrated by Neother's theorem [29] implies that the fundamental driver for PEP can actually be traced back to symmetry in nature itself. Where symmetry itself came from or why the primordial singularity from classical general relativity was present in the first place is not addressed and potentially cannot even be addressed further in this manner insofar as either any testable or repeatable observation might be offered. This assumes symmetry itself is irreducible as might be the singularity.

\section{Conclusions}

By imposing the initial singularity from General Relativity to exist at the Planck scale when $t=0$, sequential quantum transitions in Planck time intervals result in an inflationary expansion with an arbitrary number of starting fermions. Particle generation rates of $\sim 1 \mathrm{E} 10$ particles per $\sim 1 \mathrm{E} 25$ Planck time intervals give rise to 1 E250 fermion particles alone being required to come into existence in the first $\sim 1 \mathrm{E}-20$ s. After this, gluon, photon, and graviton effects (assumed to propagate at light speed) just start to take effect and attenuate the process.

The resultant effects from these basic standard model and relativistic assumptions give rise to a mechanistic expectation of observable cosmology in terms of homogeneity and isotropy on large scales while maintaining equivalent phase space dynamics in regions formed outside the horizon of comparable disjoint regions. In this way, the contrived inflaton is not required to explain big bang cosmology but rather standard model physics, constrained at the Planck scale.

\section{Acknowledgements}

Special thanks are extended to Dr. Prof. Peter Fisher of the MIT physics department and to Oak Ridge National Laboratory for support and encouragement to think outside the box. This work partially paid for by the Nuclear Regulatory Commission grant NRC-HQ-84-14-G-0059.

\section{Conflicts of Interest}

The author declares no conflicts of interest regarding the publication of this paper. 


\section{References}

[1] Sato, K. and Yokoyama, J. (2015) International Journal of Modern Physics D, 24, 1-47. https://doi.org/10.1142/S0218271815300256

[2] Uzan, J.P. (2015) Comptes Rendus Physique, 16, 875-890. https://doi.org/10.1016/j.crhy.2015.08.001

[3] Turok, N. (2002) Classical and Quantum Gravity, 19, 3449-3467. https://doi.org/10.1088/0264-9381/19/13/305

[4] Battefeld, D. and Peter, P. (2015) Physics Reports, 571, 1-66. https://doi.org/10.1016/j.physrep.2014.12.004

[5] Lolley, M. and Peter, P. (2015) Comptes Rendus Physique, 16, 1038-1047. https://doi.org/10.1016/j.crhy.2015.08.009

[6] Qui, T. and Want, Y.T. (2015) Journal of High Energy Physics, 4, 130.

[7] Klinkhamer, F.R. (2012) Physical Review D, 85, Article ID: 023509. https://doi.org/10.1103/PhysRevD.85.016011

[8] Bessada, D., Kinney, W.H., Stojkovic, D. and Wang, J. (2010) Physical Review D, 81, Article ID: 043510. https://doi.org/10.1103/PhysRevD.81.043510

[9] Kragh, H.S. (2006) Studies in History and Philosophy of Modern Physics, 37, 726-737. https://doi.org/10.1016/j.shpsb.2006.04.004

[10] Moffat, J.W. (2016) European Physical Journal, 76, 130. https://doi.org/10.1140/epjc/s10052-016-3971-6

[11] Alexander, W. (2015) International Journal of Modern Physics A, 30, Article ID: 1530024. https://doi.org/10.1142/S0217751X15300240

[12] Lidsey, J.E., Wands, D. and Copeland, E.J. (2000) Physics Reports, 337, 343-492. https://doi.org/10.1016/S0370-1573(00)00064-8

[13] Creminelli, P., Nicols, A. and Trincherini, E. (2010) Journal of Cosmology and Astroparticle Physics, 11, 1-25. https://doi.org/10.1088/1475-7516/2010/11/021

[14] Das, S. (2015) International Journal of Modern Physics D, 24, 1-8.

[15] Hollands, S. and Wald, R.M. (2002) General Relativity and Gravitation, 34, 20432055. https://doi.org/10.1023/A:1021175216055

[16] Poplawski, N.J. (2010) Physics Letters B, 694, 181-185. https://doi.org/10.1016/j.physletb.2010.09.056

[17] Capozziello, S., Nojiri, S. and Odontisov, S.D. (2006) Physics Letters B, 632, 597-604. https://doi.org/10.1016/j.physletb.2005.11.012

[18] Hossain, M.W., Myrzakulov, R., Samy, M. and Saridakis, E.N. (2015) International Journal of Modern Physics D. Gravitation; Astrophysics and Cosmology, 24, 1-53. https://doi.org/10.1142/S0218271815300141

[19] Nojiri, S. and Odintsov, S.D. (2008) Physics Letters B, 659, 821-826.

[20] Ellis, G.F.R. (1984) Annual Review of Astronomy and Astrophysics, 22, 157-184. https://doi.org/10.1146/annurev.aa.22.090184.001105

[21] Ragazzoni, R., Turatto, M. and Gaessler, W. (2003) The Astrophysical Journal, 587, L1-L4. https://doi.org/10.1086/375046

[22] Boyanovsky, D., de Vega, H.J. and Schwarz, D.J. (2006) Annual Review of Nuclear and Particle Science, 56, 441-500.

https://doi.org/10.1146/annurev.nucl.56.080805.140539

[23] Kaplan, I.G. (2017) The Pauli Exclusion Principle: Origins, Verifications and Applications. John Wiley \& Sons, West Sussex. https://doi.org/10.1002/9781118795309 
[24] Carroll, S.M., Press, W.H. and Turner, E.L. (1992) Annual Review of Astronomy and Astrophysics, 30, 499-542.

https://doi.org/10.1146/annurev.aa.30.090192.002435

[25] Lattimer, J.M. (2012) Annual Review of Nuclear and Particle Science, 62, 485-515. https://doi.org/10.1146/annurev-nucl-102711-095018

[26] Griffiths, D. (1987) Introduction to Elementary Particles. John Wiley \& Sons, New York. https://doi.org/10.1002/9783527618460

[27] Phillips, T.J. (2016) Nature, 529, 294-295. https://doi.org/10.1038/529294a

[28] Goradia, K.K., Memon, T., Kanjarpane, S.R., Atwal, A., Agrawal, D., Jadhav, K., Gandhi, D., et al. (2021) Comprehensive Review of Physics of Gravitational Waves and Functional Elements of LIGO.

Noether, E. (1918) Invariante Varlationsprobleme. Nachr. d. König. Gesellsch. d. Wiss. zu Göttingen, Math-Phys. Klasse, 235-257.

[29] Carvalho, J.C. (1995) International Journal of Theoretical Physics, 34, 2507-2509. https://doi.org/10.1007/BF00670782

[30] Giovannini, M. and Shaposhnikov, M.E. (1998) Physical Review Letters, 80, 22. https://doi.org/10.1103/PhysRevLett.80.22 